\title{
Subcycle Extreme Nonlinearities in GaP Induced by an Ultrastrong Terahertz Field
}

\author{
Carlo Vicario, ${ }^{1}$ Mostafa Shalaby, ${ }^{1}$ and Christoph P. Hauri ${ }^{1,2}$ \\ ${ }^{1}$ Paul Scherrer Institute, SwissFEL, 5232 Villigen PSI, Switzerland \\ ${ }^{2}$ Ecole Polytechnique Federale de Lausanne, 1015 Lausanne, Switzerland
}

(Received 20 November 2016; published 21 February 2017)

\begin{abstract}
We report on the experimental observation of extreme laser spectral broadening and a change in optical transmission in gallium phosphite induced by $25 \mathrm{MV} / \mathrm{cm}$ terahertz (THz) single-cycle internal field. Such intense $\mathrm{THz}$ radiation leads to twofold transient modifications of the optical properties in the electrooptical crystal. First, the electric field provokes extensive cross-phase modulation via the $\chi^{(2)}$ and $\chi^{(3)}$ nonlinearities on a copropagating 50 fs near infrared laser pulse which turns into $500 \%$ spectral broadening. Second, we observe an instantaneous change of the optical transmission occurring at the THz field which is alleged to interband Zener tunneling and charge carrier density modification by impact ionization turning the semiconductor in a metal-like transient state. The presented scheme displays a pathway to coherently control the optical properties of semiconductors on an ultrafast time scale by a strong $\mathrm{THz}$ field.
\end{abstract}

DOI: 10.1103/PhysRevLett.118.083901

Covering the energy range from a fraction of an meV to about $40 \mathrm{meV}(0.1-10 \mathrm{THz})$, terahertz $(\mathrm{THz})$ radiation directly probes charged quasiparticles, free carriers, polarons, excitons, phonon resonances, and intraband transitions in low-dimensional systems. The pump-probe scheme enables us, in addition, to visualize the off-equilibrium evolution of these excitations at a subpicosecond time scale [1-10]. The very recent advent of ultrastrong $\mathrm{THz}$ fields up to $83 \mathrm{MV} / \mathrm{cm}$ and 28 Tesla $[9,10]$ is considered the forthcoming mean to precisely control electronic and magnetic ultrafast dynamics, to drive novel highly nonlinear processes in condensed matter, to accelerate electron to relativistic energy [11-19], and to prepare quasistationary states in solids. So far, a THz-driven nonlinear $\chi^{(2)}$ process has been demonstrated in ZnTe to induce crossphase modulation (XPM) and spectral broadening on a laser pulse. However, the low $\mathrm{THz}$ field available at that time resulted in a minor spectral broadening and modifications only [20,21].

In this Letter, we report on a nonlinear optical response in an electro-optical (EO) active medium which is introduced by a single-cycle $\mathrm{THz}$ with internal field strength up to $25 \mathrm{MV} / \mathrm{cm}$. Exploring for the first time this ultrastrong field regime, we show that the optical spectrum of the femtosecond laser probe is changed spectacularly as the optical properties of the EO material become a function of the $\mathrm{THz}$ field amplitude and phase. The THz electric field drives nonlinear XPM via the Pockels and the Kerr effects. In addition, we report that the $\mathrm{THz}$ field leads to a prompt distortion of the electronic band structure which results in a subcycle time scale modification of the optical transmission and electric conductivity. As a model for the present study, we chose undoped gallium phosphite $(\mathrm{GaP})$. GaP is a crystal with a cubic orientation which is widely used for
$\mathrm{THz}$ metrology based on its linear EO response at a weak field $(\ll 10 \mathrm{kV} / \mathrm{cm})$. At the ultrastrong field regime ( $>10 \mathrm{MV} / \mathrm{cm}$ ), however, the EO response turns highly nonlinear, which manifests itself with the strong modification of the optical properties of the GaP.

The experimental outline is depicted in Fig. 1. A singlecycle ultrastrong $\mathrm{THz}$ and a laser probe are focused on a $50 \mu \mathrm{m}$ thin (110) GaP crystal. In the present configuration, the $\mathrm{THz}$ field period is long (250 fs) compared to the near infrared laser pulse (50 fs) and provides thus a quasistatic modification of the optical properties over the duration of the probe. The $\mathrm{THz}$ and the optical beam are linearly polarized along the axis for maximum EO response. The $\mathrm{THz}$ pulses of maximum energy of $50 \mu \mathrm{J}$ and spectral content in the frequency between 0.1 and $5 \mathrm{THz}$ are produced by optical rectification in Diethylaminosulfur trifluoride (DAST) $[3,9,10]$. The radiation is focused at its

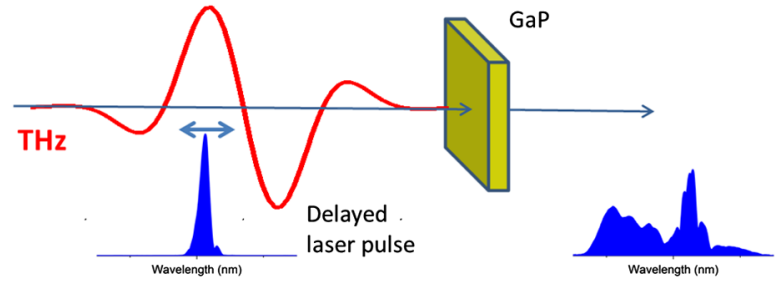

FIG. 1. Experimental setup: the $50 \mathrm{fs}, 800 \mathrm{~nm}$ laser copropagates with a strong single-cycle $\mathrm{THz}$ of $40 \mathrm{MV} / \mathrm{cm}$ in a $50 \mu \mathrm{m}$ thin GaP crystal (110). The crystal is an electro-optical active semiconductor with a $2.33 \mathrm{eV}$ band gap, larger than the probe $(1.55 \mathrm{eV})$ and $\mathrm{THz}(0.4-20 \mathrm{meV})$ photon energy. The optical properties of the $\mathrm{GaP}$ are modified by the $\mathrm{THz}$ electric field. This turns into spectral broadening of the probe and a change of optical transmission. The temporal evolutions of these phenomena are visualized as a function of the delay of the optical probe. 
physical limit to a lambda cubic volume for the realization of the maximum field strength [9]. The linear $\chi^{2}$ electrooptical effect (Pockels) is typically used for $\mathrm{THz}$ field reconstruction by electro-optical sampling (EOS) $[22,23]$. At a low amplitude, the $\mathrm{THz}$ electric field $E_{\mathrm{THz}}(t)$ simply induces a linear, time-dependent, change of the refractive index which is probed by a delayed short laser pulse. The phase retardation $\Delta \varphi(t)$ experienced by the probe and integrated over the electro-optical crystal length $L$ is proportional to $E_{\mathrm{THz}}(t)$ and can be expressed as $\Delta \varphi$ $(t)=\pi L E_{\mathrm{THz}}(t) / V_{\lambda / 2}$, where the half wave voltage $V_{\lambda / 2}$ depends on the characteristics of the electro-optical medium.

The linear relation between $\Delta \varphi(t)$ and $E_{\mathrm{THz}}(t)$ is used in the experiment for the $\mathrm{THz}$ field reconstruction, as shown in Fig. 2(a). $E_{\mathrm{THz}}(t)$ has a single-cycle shape and peak field amplitude up to a record-high value of $24 \mathrm{MV} / \mathrm{cm}$. This is the effective internal field propagating in $\mathrm{GaP}$ including the Fresnel losses at the crystal surface. The field strength is calibrated against the third-order optical birefringence in a air and diamond sample as reported in Ref. [24].

As $\mathrm{THz}$ fields exceed several $\mathrm{MV} / \mathrm{cm}$, the electrooptical properties of the GaP undergoes an instantaneous and spectacular modification. This behavior is manifested in the probe beam, which experiences a huge and prompt
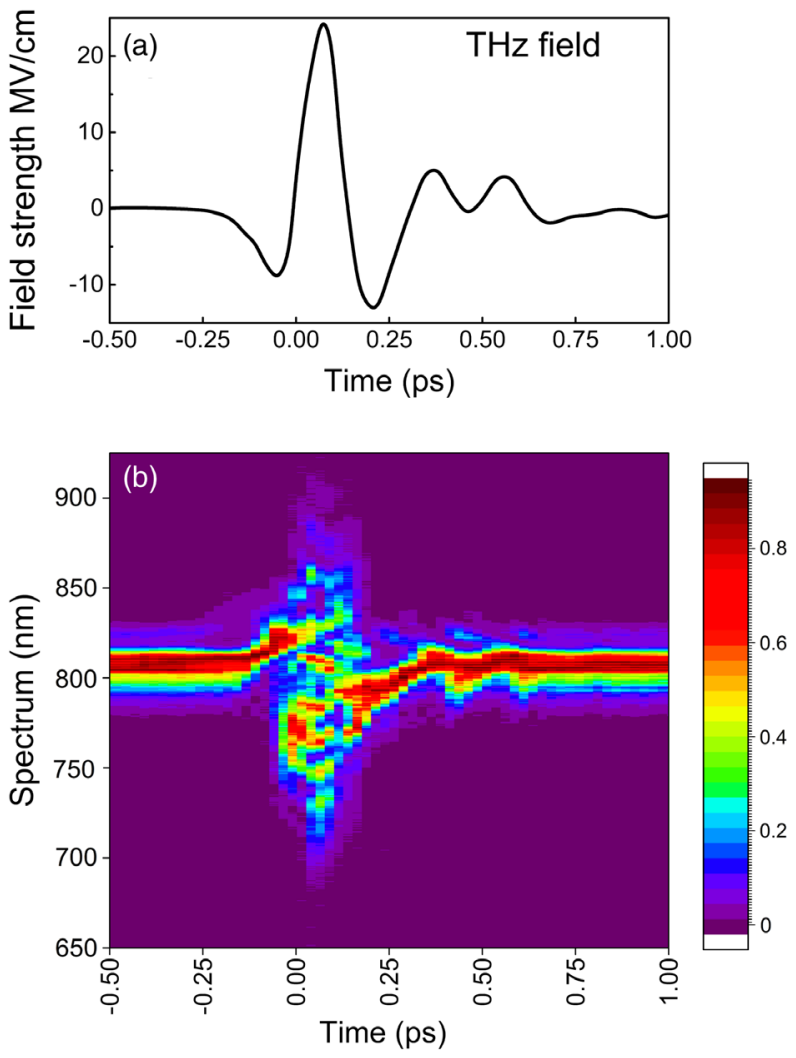

FIG. 2. (a) THz electric field reconstructed by electro-optical sampling. (b) Giant THz-induced XPM gives rise to strong spectral modification of a laser pulse. The spectrum of the probe pulse is shown as a function of the delay between the optical and $\mathrm{THz}$ field. spectral broadening with the main driver being the crossphase modulation. Shown in Fig. 2(b) is the evolution of the laser spectrum caused by the THz field. XPM is the optical phase change experienced by the probe beam in $\mathrm{GaP}$ and induced by the strong copropagating $\mathrm{THz}$ beam. From our measurements, it turns out that the spectral modifications are directly related to the $\mathrm{THz}$ field oscillations shown in Fig. 2(a). When the probe overlaps with the $\mathrm{THz}$, a strong spectral broadening occurs as a function of the delay $\Delta \tau$. At the maximum $\mathrm{THz}$ field $(\Delta \tau \approx 50 \mathrm{fs}$ ), the laser spectrum is broadened by more than a factor of 5 , to a maximum width of $190 \mathrm{~nm}(-10 \mathrm{~dB}$ level). This extreme spectral modification and broadening originates from the nonlinear change of the refractive index by giant THz-induced XPM. At delays $\Delta \tau$ earlier than the THz pulse $(\Delta \tau<-0.25 \mathrm{ps})$, the natural laser spectrum is preserved, indicating the absence of self-induced spectral distortions and self-phase modulation. It is worth noting that the instantaneous spectral broadening takes place on a subcycle time scale of $E_{\mathrm{THz}}(t)$ and supports the formation of sub-10-fs pulses. For $\Delta \tau>750 \mathrm{fs}$, after the $\mathrm{THz}$ pulse the XPM process breaks down. Consequently, the probe spectrum resumes the initial narrow laser spectrum.

In the present experiment, we show that XPM is mediated via $\chi^{(2)}$ (Pockels) and $\chi^{(3)}$ (Kerr) corresponding to phase retardation proportional to the field and the intensity of the $\mathrm{THz}$ pulse. This is different from recent studies where THz-driven XPM was demonstrated through second-order coupling only and at low fields [20,21]. For physical insights, it is useful to write the index of refraction as $n=n_{1}+n_{2}+n_{3}$, where the second term accounting for the Pockels effect is $\Delta n_{2}=\chi^{(2)} E_{\mathrm{THz}}(t) / n_{1}$ and the thirdorder Kerr term depends on the THz intensity as $\Delta n_{3}=$ $3 \chi^{(3)}\left|E_{\mathrm{THz}}(t)^{2}\right| / 2 n_{1}$. The phase retardation accumulated in the EO crystal can be decomposed in the Taylor expansion [20]: $\Delta \varphi(t)=\varphi_{0}+d \varphi_{0}^{\prime}\left(t-t_{0}\right)+d \varphi_{0}^{\prime \prime}\left(t-t_{0}\right)^{2}+\ldots$. Here $\varphi_{0}$ represents the phase retardation proportional to $E_{\mathrm{THz}}(t)$ and is measured in conventional EOS. The second term of the series corresponds to the wavelength shift, while the third one induces spectral broadening. For the Pockels effect only, the spectral modifications depend directly on $E_{\mathrm{THz}}(t)$ resulting in a frequency shift at the zero crossing (when the second term of the above expansion is maximum) and broadening due to quadratic phase modulation at the crest and the troughs of the $E_{\mathrm{THz}}(t)$. At the maximum fields employed in the present experiment, the refractive index varies in a complex way, as the third-order term in the Taylor expansion becomes important. Because of the simultaneous occurrence of the Pockels and Kerr effects, the phase retardation depends on the field and intensity of the $\mathrm{THz}$ stimulus. Therefore, the spectral modifications become much more pronounced.

According to the above time-shift model, the spectral broadening presented in Fig. 2(b) is caused by quadratic phase modulation and occurs at the crest and the troughs of 

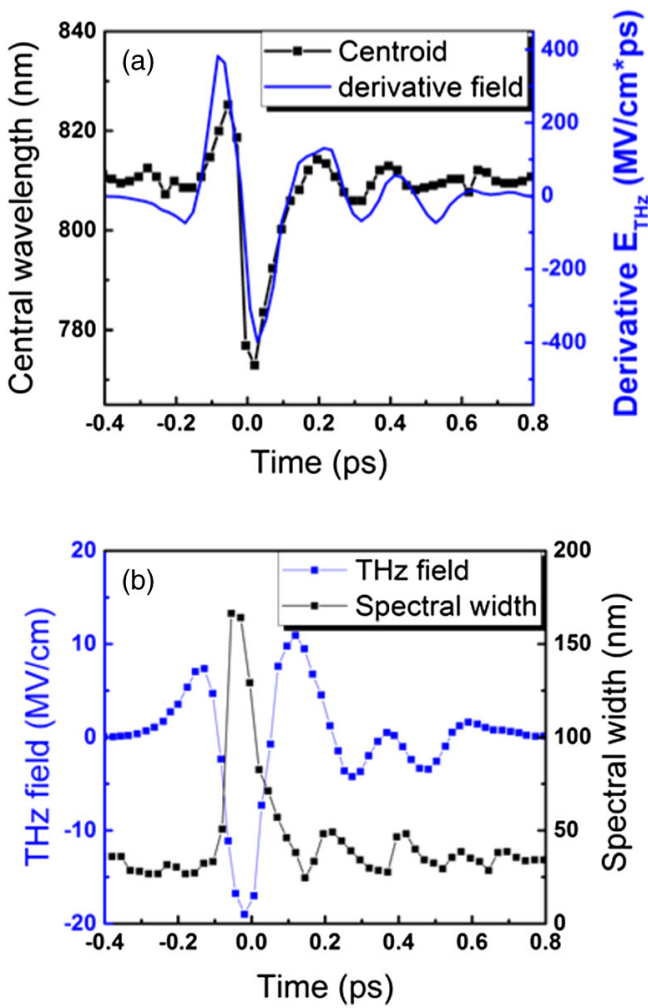

FIG. 3. Laser spectral centroid and $-10 \mathrm{~dB}$ width are well approximated by the derivative and the amplitude of the $\mathrm{THz}$ waveform. (a) The central wavelength of the probe is blueshifted by more than $40 \mathrm{~nm}$, while (b) the spectral width is broadened by almost a factor of 5 when overlapping with the maximum $\mathrm{THz}$ field.

the electric field, while the wavelength shift is proportional to the first derivative of $E_{\mathrm{THz}}(t)$. Even though the simultaneous occurrence of the Kerr and Pockels effects makes the relation between the spectral modification and the $\mathrm{THz}$ waveform more complex, it is possible to correlate the spectral broadening and spectral centroid shift induced by the XPM with $E_{\mathrm{THz}}(t)$ and $d / d t E_{\mathrm{THz}}(t)$, respectively. The results are reported in Fig. 3. In excellent agreement with the time-shift model, there is a clear correlation between the wavelength centroid (black curve) and the first derivative of the THz field [Fig. 3(a), blue curve]. The centroid shifts predominantly towards the short wavelengths. As shown in Fig. 3(b), the spectral broadening occurs over the time scale of $E_{\mathrm{THz}}(t)$ (blue curve) and gives rise to a maximum spectral width at $-10 \mathrm{~dB}$ (black curve) of $500 \%$ at the peak of the field. We note that after the interaction the spectral parameters (centroid and bandwidth) instantly follow the initial laser characteristics.

In Fig. 4, the spectral modifications of the probe are presented in dependence of the $\mathrm{THz}$ peak field at maximum amplitude $E_{\max }$. For this measurement, the THz intensity is controlled by means of a polarizer and analyzer which allowed an extinction of the field down to a fraction of a few percent. This attenuation scheme was cross-calibrated with respect to the EOS signal. The initial laser spectrum
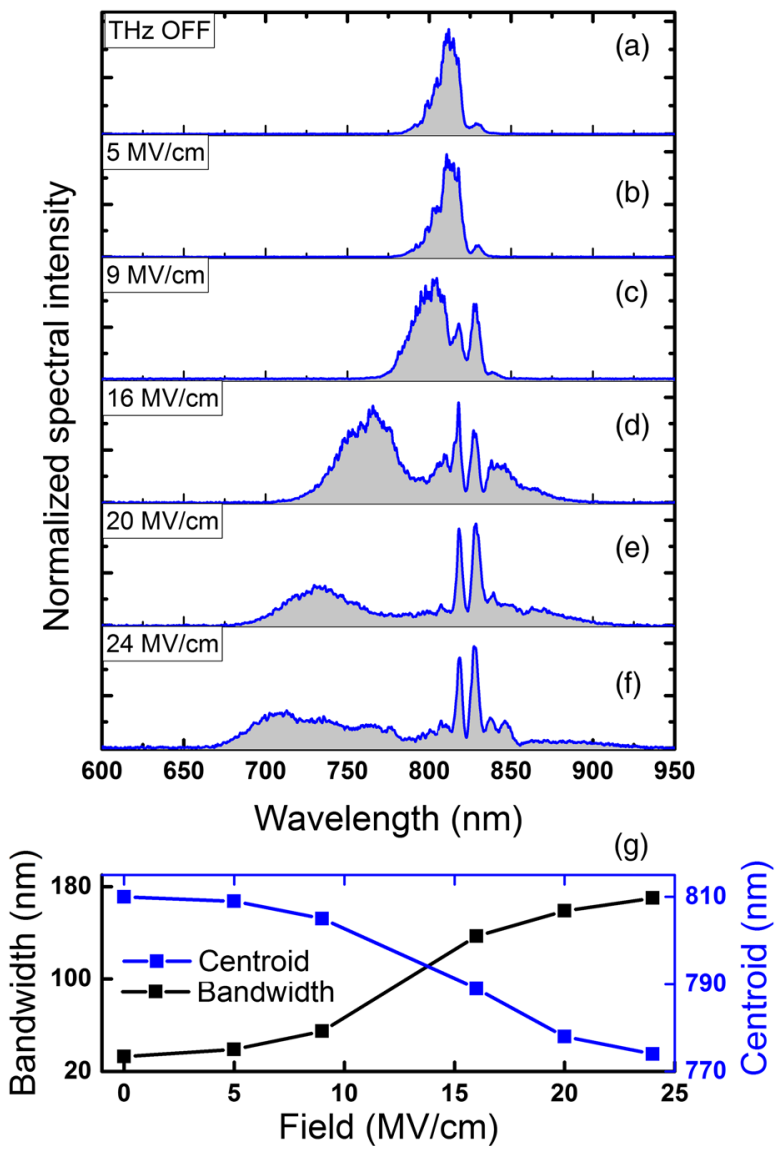

FIG. 4. (a) The original laser spectrum recorded at the $\mathrm{THz}$ peak field amplitude is progressively broadened (b)-(f) by XPM for increasing $\mathrm{THz}$ field strengths. (g) The $-10 \mathrm{~dB}$ spectral width (black curve) and centroid (blue curve) are shown as a function of the $\mathrm{THz}$ peak field.

shown in Fig. 4(a) is not significantly modified by XPM up to a $\mathrm{THz}$ field of $5 \mathrm{MV} / \mathrm{cm}$ [Fig. 4(b)]. However, when the field exceeds $9 \mathrm{MV} / \mathrm{cm}$ [Figs. 4(c)-4(e)], the spectral width rapidly increases. Finally, for a field above $20 \mathrm{MV} / \mathrm{cm}$ [Figs. 4(e) and 4(f)], the spectral modifications reach the maximum. Shown in Fig. 4(g) (black curve) is the $-10 \mathrm{~dB}$ spectral width as a function of the peak $\mathrm{THz}$ field. A noticeable blueshift of the laser spectrum is observable as a function of the field strength, [blue curve in Fig. 4(g)]. The prevalence of spectral shift towards shorter wavelengths is associated to a multiplication of carriers in the conduction band, and its physical origin is discussed later. Similar to plasma-related spectral blueshifting observed in gas, the delocalized conduction band electrons are expected to contribute to a spectral broadening towards higher frequencies. Such a strong blueshift is observed both in Fig. 3(a) and in Fig. 4(g).

In order to disentangle the contributions of the Pockels and the Kerr effects, we study the XPM as a function of the GaP crystal orientation. In these measurements, the $\mathrm{THz}$ field and the probe laser carry linear and parallel polarizations. Because of symmetry considerations, the second-order susceptibility varies with the azimuthal 
Crystal orientation (deg)

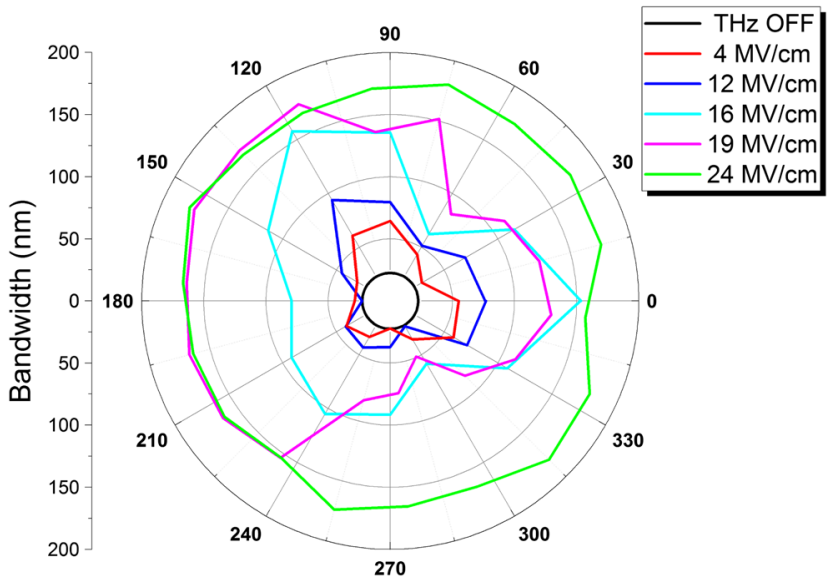

FIG. 5. Maximum spectral width at $-10 \mathrm{~dB}$ recorded as a function of the $\mathrm{GaP}$ orientations. The different curves refer to different $\mathrm{THz}$ field strengths.

crystal angle. As reported in Ref. [25], the $\chi^{(2)}$-related electro-optical signal periodically vanishes for a sequence of crystal orientations showing three positive and negative maxima for a full $360^{\circ}$ crystal turn. A similar angular dependence on the probe is thus expected for spectral modifications driven by $\chi^{(2)}$-only processes, while $\chi^{(3)}$ only broadening processes do not depend on the crystal orientation. For further insights, we recorded the $-10 \mathrm{~dB}$ spectral width at the peak of $E_{\mathrm{THz}}(t)$ as a function of the GaP angle for three different field strengths (Fig. 5). The black curve shows that without the XPM driven by the $\mathrm{THz}$ field the spectral width is independent of the orientation of the crystal. The maximum electro-optical effect is expected at $0^{\circ}$, whereas the EO effect should vanish at $60^{\circ}$ (crystallographic axis $[0,0,1]$ ).

Correspondingly, the XPM caused by $\chi^{(2)}$ is expected to vanish at this angle, while, as shown in the graph, the broadening is increasing as the $\mathrm{THz}$ field becomes larger. Moreover, for $180^{\circ}$ inversion, $\chi^{(2)}$ changes sign while $\chi^{(3)}$ does not. This is consistent with the results at a low field indicating that the combination of Pockels and Kerr effects is constructive for angles $\left(0^{\circ}, 120^{\circ}\right.$, and $\left.240^{\circ}\right)$ and destructive for the opposite directions. At the maximum field of $24 \mathrm{MV} / \mathrm{cm}$ (green curve), moreover, the spectral broadening becomes insensitive to the crystal angle due to the predominance of the $\chi^{(3)}$, which is invariant to the crystal orientation.

We point out that negligible changes in divergence and reduced polarization rotation (a few percent) were observed for the spectrally broadened laser beam.

Under the influence of the $\mathrm{THz}$ field, not only the refractive index of the $\mathrm{GaP}$ is rapidly modified but also its conductivity. The strong $\mathrm{THz}$ transient provokes, an instantaneous change of the material properties from semiconductorlike towards metal-like. Consequently, the transmission of the below-band-gap laser probe (photon energy of $1.55 \mathrm{eV}$ and band gap of $2.23 \mathrm{eV}$ ) is reduced on the $\mathrm{THz}$ (a)

Time (ps)

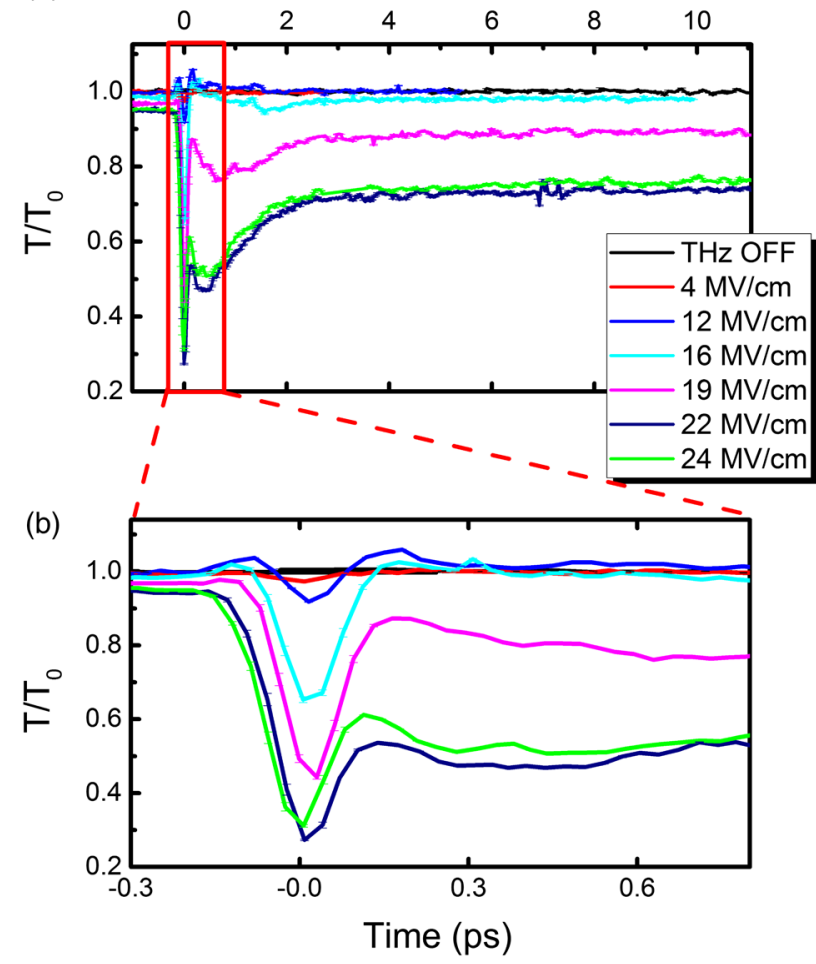

FIG. 6. Change in the optical transmission in $50 \mu \mathrm{m} \mathrm{GaP}$ in a strong THz field regime. (a) and (b) show the temporal evolution of the transmission on a long and fast time scale induced by the single-cycle $\mathrm{THz}$ field peaked at zero delay.

subcycle time scale with an increase of the reflectivity [Figs. 6(a) and 6(b)]. At the THz maximum internal field of $24 \mathrm{MV} / \mathrm{cm}$, the near infrared transmission is reduced by $73 \%$. In the presence of an intense electromagnetic field, multiphoton or tunneling ionization may occur depending on the driving frequency and strength. The Keldysh parameter $\gamma_{k}$ [26] is a quantitative indicator of the ionization regime: $\gamma_{k} \gg 1$ indicates a multiphoton and $\gamma_{k} \ll 1$ a tunnel. The THz-driven increase of conductivity is attributed to a substantial modification of the GaP band structure and Zener electron tunneling. Multiphoton ionization by a THz field is excluded by the very low Keldysh parameter calculated for the experimental parameters $\left(\gamma_{k} \sim 2.6 \times 10^{-2}\right)$. Moreover, the ponderomotive energy exchange between the $\mathrm{THz}$ field and the free carriers gives rise to multiple impact ionization events with consequent large carrier multiplication. Tunneling and carrier multiplication have been reported for a direct semiconductor patterned with a gold metamaterial to enhance the $\mathrm{THz}$ field beyond $10 \mathrm{MV} / \mathrm{cm}$ [27]. The change of transmission observed in the strong-field interaction regime studied here is orders of magnitude larger than previous work on bare semiconductors [28]. It is worth noting that the presented scheme offers an important advantage over previously used field enhancement structures which are characterized by a resonant temporal response [27]. Being free of resonances, our approach provides thus a direct access 
to the time-resolved electronic and optical dynamics driven by the $\mathrm{THz}$ stimulus. We point out that losses due to absorption are negligible for an $800 \mathrm{~nm}$ pulse (Fig. 6, black curve).

The measurements indicate the coexistence of fast and retarded carrier dynamics. The fast one occurs on a temporal scale comparable to the driving $\mathrm{THz}$ field intensity. In the ultrastrong field regime, the quick reduction of the optical transmissivity at the $\mathrm{THz}$ peak is followed by a slow recovery. This double behavior can be qualitatively explained by the interplay of electronic tunneling, carrier multiplication, and thermal effects. The fast dynamics is alleged to Zener tunneling and successive impact ionization while the slow dynamics to subsequent thermalization of the semiconductor, similar to Ref. [29]. The indirect band structure of the GaP does not allow the quick recombination of the electron-hole pairs. It is worth noting that the recovery of the $\mathrm{GaP}$ transmission takes place over a time scale longer than the delay range accessible in the experiment. A closer look at the transmission before the main pulse indicates that for fields $>15 \mathrm{MV} / \mathrm{cm}$ the transmission is not fully recovered after $10 \mathrm{~ms}$, corresponding to the $100 \mathrm{~Hz}$ repetition rate of the laser.

In conclusion, we demonstrate that the $\mathrm{THz}$-induced nonlinear XPM in GaP is a powerful means to control the spectral properties of ultrashort laser pulses at the sub-THz cycle. The cross-phase modulation is established through the combination of the Pockels and the Kerr effects. The magnitude of the effects presented in this study overcomes by far previous works and makes the THz-driven XPM attractive for ultrafast spectral shaping of laser pulses. In the nonperturbative regime, the electro-optical properties are accompanied by an ultrafast nonlinear transient modification of the optical conductivity induced by extreme high $\mathrm{THz}$ fields. The effect of the $\mathrm{THz}$ field on the electronic properties of semiconductors in the strong-field regime is of great interest to foster fundamental knowledge and to open new applications towards high-speed electronics.

We are grateful to Dr. Gunnar Arisholm for fruitful discussions. We acknowledge financial support from Swiss National Science Foundation (Grants No. 200021_146769, No. IZLRZ2_164051, and No. IZKSZ2_162129). M. S. is grateful to partial funding from the European Community's Seventh Framework Programme (FP7/2007-2013) under grant agreement No. 290605 (PSI-FELLOW/COFUND). C. P. H. acknowledges association to NCCR-MUST.

[1] M. Tonouchi, Nat. Photonics 1, 97 (2007).

[2] T. Kampfrath, K. Tanaka, and K. A. Nelson, Nat. Photonics 7, 680 (2013).

[3] C. Vicario, B. Monoszlai, and C. P. Hauri, Phys. Rev. Lett. 112, 213901 (2014).
[4] C. Ruchert, C. Vicario, and C. P. Hauri, Phys. Rev. Lett. 110, 123902 (2013).

[5] H. Hirori, A. Doi, F. Blanchard, and K. Tanaka, Appl. Phys. Lett. 98, 091106 (2011).

[6] F. Blanchard, X. Ropagnol, H. Hafez, H. Razavipour, M. Bolduc, R. Morandotti, T. Ozaki, and D. G. Cooke, Opt. Lett. 39, 4333 (2014).

[7] Shu-Wei Huang, E. Granados, W. R. Huang, K.-H. Hong, L. E. Zapata, and F. X. Kärtner, Opt. Lett. 38, 796 (2013).

[8] J. A. Fülöp, Z. Ollmann, Cs. Lombosi, C. Skrobol, S. Klingebiel, L. Pálfalvi, F. Krausz, S. Karsch, and J. Hebling, Opt. Lett. 22, 20155 (2014).

[9] M. Shalaby and C. P. Hauri, Nat. Commun. 6, 5976 (2015).

[10] C. Vicario, A. V. Ovchinnikov, S. I. Ashitkov, M. B. Agranat, V. E. Fortov, and C. P. Hauri, Opt. Lett. 23, 4573 (2015).

[11] C. Vicario, C. Ruchert, F. Ardana-Lamas, P. M. Derlet, B. Tudu, J. Luning, and C. P. Hauri, Nat. Photonics 7, 720 (2013).

[12] S. Fleischer, Y. Zhou, R. W. Field, and K. A. Nelson, Phys. Rev. Lett. 107, 163603 (2011).

[13] M. Shalaby, C. Vicario, and C. P. Hauri, Appl. Phys. Lett. 108, 182903 (2016).

[14] A. T. Tarekegne, K. Iwaszczuk, M. Zalkovskij, A. Strikwerda, and P. U. Jepsen, New. J. Phys. 17, 043002 (2015).

[15] M. Shalaby, C. Vicario, and C. P. Hauri, Nat. Commun. 6, 8439 (2015).

[16] O. Schubert, M. Hohenleutner, F. Langer, B. Urbanek, C. Lange, Y. Huttner, D. Golde, T. Meier, M. Kira, S. W. Koch, and R. Huber, Nat. Photonics 8, 119 (2014).

[17] M. Shalaby and C. P. Hauri, Appl. Phys. Lett. 106, 181108 (2015).

[18] M. Shalaby, C. Vicario, and C. P. Hauri, New. J. Phys. 18, 013019 (2016).

[19] E. A. Nanni, W. R. Huang, K. H. Hong, K. Ravi, A. Fallahi, G. Moriena, R. J. D. Miller, and F. X. Kaertner, Nat. Commun. 6, 8486 (2015).

[20] Y. Shen, T. Watanabe, D. A. Arena, C.-C. Kao, J. B. Murphy, T. Y. Tsang, X. J. Wang, and G. L. Carr, Phys. Rev. Lett. 99, 043901 (2007).

[21] Yuzhen Shen, G. L. Carr, James B. Murphy, Thomas Y. Tsang, Xijie Wang, and Xi Yang, Phys. Rev. A 81, 053835 (2010).

[22] X.-Y. Peng, J.-H. Teng, X.-H. Zhang, and Y.-L. Foo, J. Appl. Phys. 108, 093112 (2016).

[23] S. Casalbuoni, H. Schlarb, B. Schmidt, P. Schmuser, B. Steffen, and A. Winter, Phys. Rev. ST. Accel. Beams 11, 072802 (2008).

[24] M. Sajadi, M. Wolf, and T. Kampfrath, Opt. Express 23, 28985 (2015).

[25] P. C. M. Planken, H-K Nenhuys, H. J. Bakker, and T. Wenckebach, J. Opt. Soc. Am. B 18, 313 (2001).

[26] L. V. Keldysh, Sov. Phys. JETP 20, 1037 (1965).

[27] C. Lange, T. Maag, M. Hohenleutner, S. Baierl, O. Schubert, E. R. J. Edwards, D. Bougeard, G. Woltersdorf, and R. Huber, Phys. Rev. Lett. 113, 227401 (2014).

[28] F. Novelli, D. Fausti, F. Giusti, F. Parmigiani, and M. Hoffmann, Sci. Rep. 3, 1227 (2013).

[29] H. Hirori, K. Shinokita, M. Shirai, S. Tani, Y. Kadoya, and K. Tanaka Nat. Commun. 2, 594 (2011). 